\title{
RT-Level Route-and-Place Design Methodology for Interconnect Optimization in DSM Regime
}

\author{
Ananth Durbha \\ and Srinivas Katkoori \\ 4202, East Fowler Avenue, ENB 118, \\ Department of Computer Science and Engineering, \\ University of South Florida, Tampa, FL 33620. \\ \{durbha,katkoori\}@csee.usf.edu
}

\begin{abstract}
As the interconnect dominates in DSM regime, we propose a novel routeand-place design methodology at RT-level which strongly ties to physical design tasks. Unlike conventional place-and-route, our methodology prioritizes interconnect over devices. At the RT-level, we identify all global nets and carefully design their interconnect topology to achieve desired delay characteristics, followed by the macro-cell placement. Net clustering is carried out to account for net interdependencies as well as to derive the bounding box constraint on a net. Within a macro-cell, as the nets are local in nature, we apply place-and-route to achieve its layout. Experimental results on various datapath circuits resulted in total wirelength reduction varying from $3.7 \%$ to $24.6 \%$ with an average value of $12.4 \%$.
\end{abstract}

Keywords: Route \& Place Methodology, Interconnect Minimization, Deep Sub Micron Regime.

\section{INTRODUCTION}

Due to rapid technology scaling, technology generations in the DSM regime give us the capability to realize system-on-a-chip. The accompanied price is the dominance of the interconnect phenomena that were hitherto ignored at the micron and sub-micron technology generations. Therefore, existing CAD algorithms must be revisited and/or new CAD algorithms must be developed 
to address this paradigm shift. In the 1997 SIA NTRS [1], for example, it is projected that for $100 \mathrm{~nm}$ technology, the interconnect delay for $\mathrm{Al}$ metalization is approximately fifteen times the gate delay. This underlines the severity of the problem. The conventional place-and-route approach is not appropriate for design optimization in DSM regime as the cell placement limits the routing freedom of a net. Thus, in order to make the design methodology interconnect centered, we propose a route and place scheme so that we give priority to net routing over cell placement.

If there are no cells placed, where is one going to route the nets!! We address this problem by macro-cell clustering i.e. the cells connected by a bunch of nets are grouped together to give rise to a bounding box constraint for the nets. The clustering is based on the interdependency of nets. Those nets which have many cells in common are put in the same cluster so that the overall wirelength of each net is reasonable. Once clusters are formed, within each cluster we would synthesize the nets that satisfy the delay constraint as well as the bounding box area constraint. It is possible that clusters may share macro-cells. Thus, cluster-level placement is carried out to place the common cells between two clusters at their common boundary. Within each macro-cell, place-and-route methodology is adopted to achieve its layout, as the nets within the cell are local in nature. In summary, this approach gives highest priority to the optimization of the global net characteristics, which is key to success in DSM regime.

The rest of the paper is organized as follows: Section 2. discusses the related work. Section 3. presents the route and place design methodology in detail. Section 4. shows the experimental results. Finally, Section 5. draws conclusions and outlines future work.

\section{LITERATURE SURVEY}

Although sophisticated models for interconnect delay were developed [2] , their use in synthesis is limited due to the lack of knowledge of geometry of interconnect early enough. So synthesis tools must adopt an approach that first focussed on determining optimal plan for global wiring. Such approaches are known as wire planning.

Given a set of pre-designed modules, which may be standard cells or custom modules, and a netlist describing the connections to be made between them, the objective of module placement stage is to assign each module a unique location on the chip, so that no two modules overlap, and the design constraints are met. Wire planning [3] is one possible source for generating these constraints. There will also be design constraints imposed by the particular design style adopted.

Constructive and Iterative approach. Constructive approach try to imitate the approach taken by a human designer. But the intuitive rules of human 
designer have proven to be hard to capture into a computer program, resulting in poor quality. Iterative approach involves starting with a seed(initial placement) and improving it iteratively until closure is achieved. For large designs, number of iterations itself can be a bottleneck. Avoiding local minima is also a problem.

Stochastic approach. These algorithms try to avoid the local minima problem noted above. They allow random moves that may increase the cost function temporarily allowing for the solution to proceed in search of a "global minimum". For example simulated annealing is one such approach [4] . But this approach entails a large amount of computation which tends to be a handicap when dealing with very large circuits.

Min-Cut placement. The most common objective used during above placement process was to minimize the total interconnect length. Such algorithms often lead to non-uniform routings. This can lead to chip area not being minimized even if the wires are short.

Hence min-cut placement techniques [5, 6] were developed which seek in addition, to minimize number of nets crossing a set of horizontal and vertical cut lines crossing the chip. Shorter wirelengths are achieved by finding strongly connected groups of modules.

Timing driven placement. With interconnect delays playing an increasingly significant role in the timing closure problem, it is no longer sufficient to just guarantee routability of a particular placement but needs to satisfy length(delay) constraints. This concern led to work on timing driven placement [7] and routing [8] algorithms. These algorithms can be broadly classified as net-based [9] and path-based [10] approach. Net-based approach usually involves computing timing slack for each net and converting them into wirelength upper-bound constraints or net weights to be used in the cost function during placement. Path-based approach involves modeling path-based constraints as a mathematical programming problem.

The above approaches address the problem in a piece-wise fashion by considering point-to-point length constraints. In DSM regime, however, the entire topology of the net affects the delay. Our approach is global in nature when deriving a routing solution for a given net. The influence of the topology on the delay can be reduced by appropriate buffer insertion through isolation of the downstream capacitance.

\section{ROUTE-AND-PLACE DESIGN METHODOLOGY}

The design flow of the proposed Route-and-Place design methodology is as shown in Figure 1 The input netlist consists of instances of RT-level components such as n-bit adders, n-bit registers, m-to- 1 multiplexors, etc. We assume that the bounding box area of the leaf-cells used to build the macro-cells is 
known apriori. The netlist may be either a hand-written one or the output of a front-end synthesis tool. From the netlist, we eliminate the following global nets: power, ground, clock, and nets such as Global Reset. Specialized net topologies need to be generated for such nets. Grouping of nets that drive the same macro-cells is also carried out.

From the pre-processed netlist, net clusters are formed based on multi-net dependency. For each cluster so formed, the nets in that cluster are prioritized according to their cell-count. Following this priority, an optimum net topology is synthesized for each net. After all nets are routed, macro-cells are then placed around the nets. Within each macro-cell, the traditional place and route is carried out as the nets within a macro-cell are local in nature. Between two or more clusters macro-cells may be shared. To handle this scenario, inter-cluster placement is performed, following which an optimized layout is generated. In the following paragraphs we present a detailed discussion on each phase.

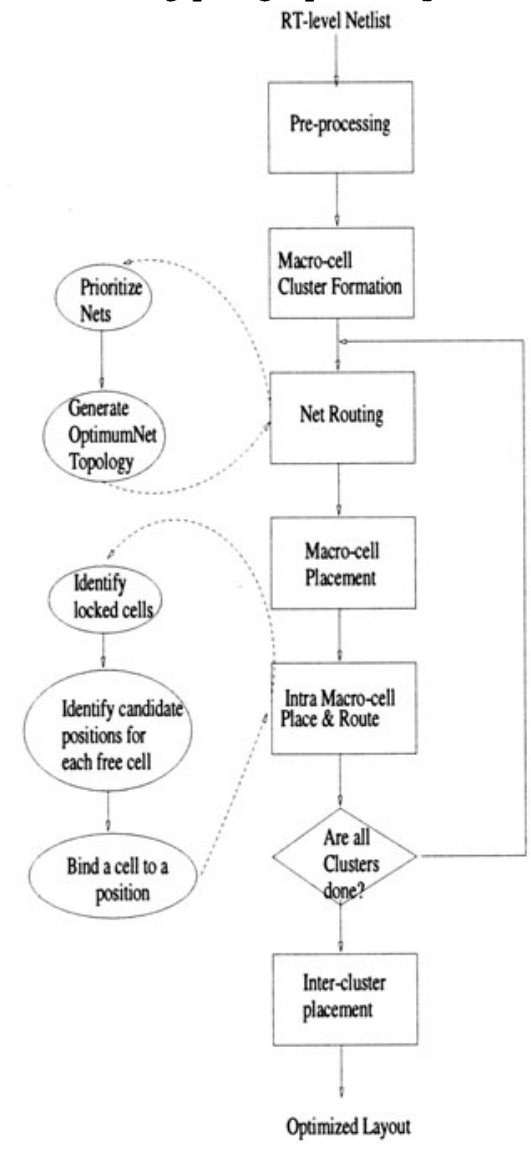

Figure 1 Route and Place Design

I. Net Clustering: Two nets are said to be interdependent provided they have at-least one common cell. The higher the number of common cells, higher the degree of interdependency. Strongly interdependent nets must be routed in the same region in order to find "good" interconnect topologies for all the nets in question. Net clustering is carried out based on the net interdependencies. We employ clique partitioning algorithm to form the clusters.

From the input netlist, a net interdependency graph, $G$, is constructed where each node represents a net and an edge exists between two nodes if and only if the nets represented by the nodes are interdependent. Each edge is weighted with a weight equal to the number of common cells between the nets. A modified version of clique partitioning algorithm proposed by Tseng and Siewiorek [11] is applied on $G_{d u a l}$, the dual of $G$. The main modification to the original algorithm is to handle a weighted input graph. 
The advantage of applying clique partitioning on $G_{d u a l}$ is that it not only yields cliques of nets but also information as to which nets within the clique needs to closer. In other words, it yields clusters that are hierarchical in nature.

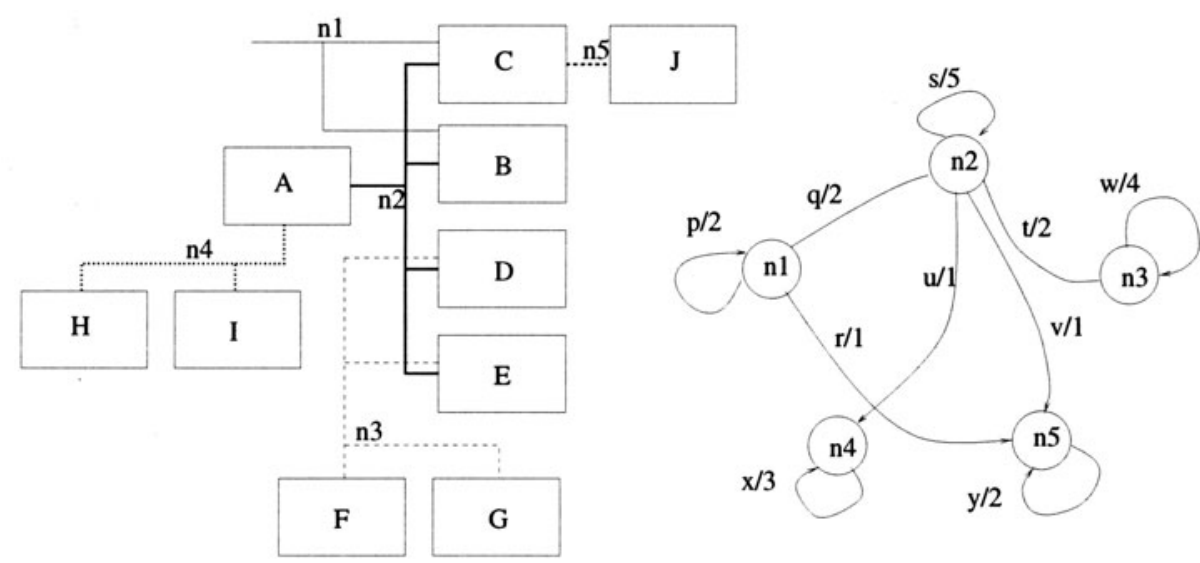

Figure (a)

Figure (b)

Figure 2 (a) A Simple netlist with five nets connecting ten RTL modules; (b) Net interdependency graph

For a simple RTL netlist shown in Figure 2(a) $G$ and $G$ dual are shown in Figure 2(b) and Figure 3. Clique partitioning on $G$ dual yields minimum number of maximum weighted cliques. The following clique set is obtained from $G_{\text {dual }}: C=\{\{\mathrm{b}, \mathrm{d}, \mathrm{e}, \mathrm{f}, \mathrm{g}\},\{\mathrm{a}, \mathrm{c}\},\{\mathrm{i}\},\{\mathrm{j}\},\{\mathrm{h}\}\}$.

On expanding the clique set into net clusters, we obtain the following: $C^{\prime}=\{\{\mathrm{n} 1, \mathrm{n} 2, \mathrm{n} 3, \mathrm{n} 4, \mathrm{n} 5\},\{\mathrm{n} 1, \mathrm{n} 5\},\{\mathrm{n} 3\},\{\mathrm{n} 4\},\{\mathrm{n} 5\}\}$. As clique partitioning is performed on $G_{d u a l}$, a net may occur in more than one cluster. In a given cluster, we can group nets into sub-clusters that identify the nets that are desired to be routed very closely in the same region. A sub-cluster in a cluster arises under the following conditions:

(1) An identical cluster exists by itself.

(2) An identical sub-cluster occurs in one or more other cliques.

The reduction of $C^{\prime}$ gives rise to $C^{\prime \prime}=\{\{\{\mathrm{n} 1, \mathrm{n} 5\}, \mathrm{n} 2, \mathrm{n} 3, \mathrm{n} 4\}\}$, where $\{\mathrm{n} 1, \mathrm{n} 5\}$ is a sub-cluster that occurs by itself. Thus, we obtain one hierarchical cluster with two levels.

II. Routing: In [12] it is shown that both the total wirelength (i.e., the total interconnect capacitance) as well as the interconnect topology will dictate the interconnect delay. Our methodology gives near complete freedom 


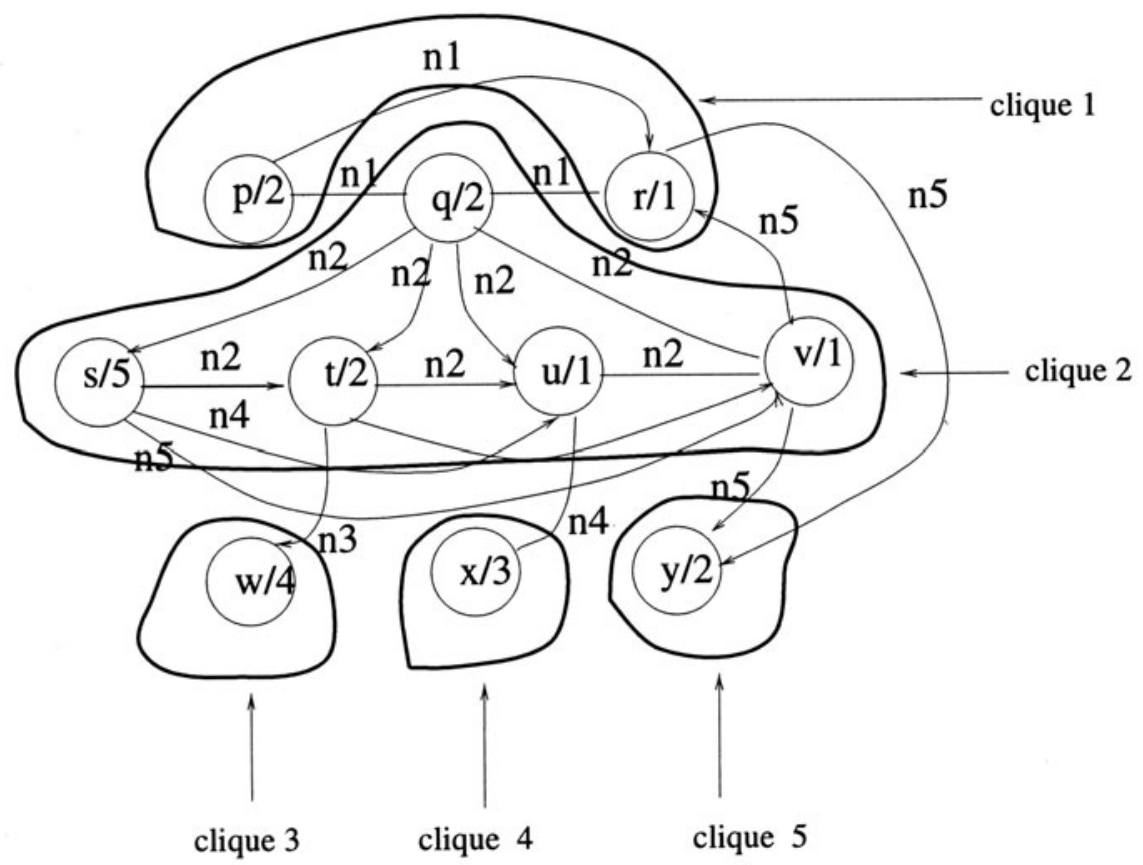

Figure 3 Dual of the graph shown in Figure 1.2(b)

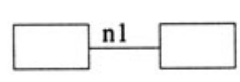

(a)

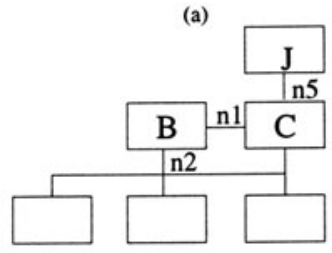

(c)

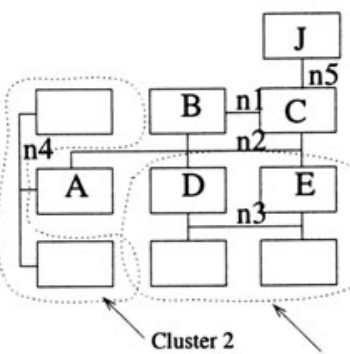

(e)

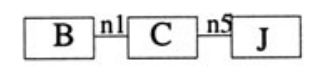

(b)

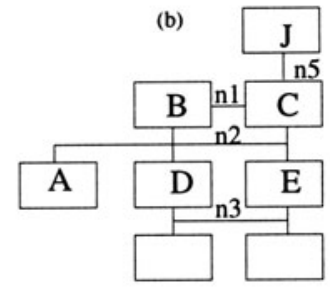

(d)

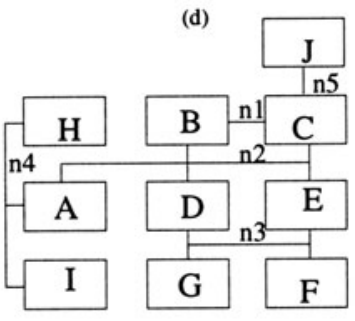

(f)

Figure 4 (a)-(e) Various stages of routing the cluster (f) Final layout 
to optimize both factors for every global net. In this work, we focus on total wirelength minimization. Thus, given a cluster, for every net belonging to the cluster, we aim to obtain an optimum interconnect topology in terms of its total wirelength. We assume that the delay is linearized by repeater insertion and therefore the wirelength is a reasonable delay metric. The delay of a long line that is "quadratic" in nature can be canonically converted into "near-linear" delay by buffer insertion, as a buffer isolates the "downstream" capacitance [13]. Optimal buffer insertion [14] algorithms exists in the literature that can be employed for this purpose.

Work is in progress to compare the actual delays by employing accurate timing analysis tools such as moment-based timing tools [15] and HSPICE.

The generation of a routing solution begins from the innermost sub-cluster(s) and works outwards. For eg., in case of $C^{\prime \prime}$, first we generate a routing solution for $\{n 1, n 5\}$ and then continue with nets $n 2, n 3$, and $n 4$. For those nets which are at the same level within a cluster, we given preference to larger nets i.e. nets with higher cell count. As the routing solution evolves, macro-cells get locked into certain positions and the end all common macro-cells are locked. Thus a partial placement solution also evolves as a side-effect of this phase. Re-positioning of macro-cells is carried out with the objective of making the layout as square as possible.

Figure 4 shows how the routing solution for the cluster $C$ " evolves. The sub-cluster $\{\mathrm{n} 1, \mathrm{n} 5\}$ within $C^{\prime \prime}$ is routed first. Figure 4(a) shows a simple topology for net $\mathrm{n} 1$ with two possible locations for the macro-cells belonging to $n 1$. Figure 4(b) depicts the addition of net $n 5$ to net $n 1$. This fixes the positions of the macro-cells $\mathrm{B}, \mathrm{C}$, and $\mathrm{J}$. Between the two possible candidates $\mathrm{n} 2$ and $\mathrm{n} 3$, we randomly pick $\mathrm{n} 2$. With macro-cells $\mathrm{B}$ and $\mathrm{C}$ already placed, we construct a net topology for $\mathrm{n} 2$ as shown in Figure 4(c). There are three possible cell positions that can be occupied by the remaining cells on the net, namely $\mathrm{A}, \mathrm{D}$, and $\mathrm{E}$. Routing of net $\mathrm{n} 3$ necessitates the placement of macrocells $\mathrm{D}$ and $\mathrm{E}$ that are shared with net $\mathrm{n} 2$. Thus, macro-cells $\mathrm{D}$ and $\mathrm{E}$ are placed as shown in Figure 4(d). Finally, net $n 3$ is routed as shown in Figure 4(e).

III. Macro-cell Placement: In the routing solution obtained in the previous phase, the placement of unbound macro-cells is carried out. Generate pin location constraints for each macro-cell. Within a macro-cell (eg. an n-bit adder) since all the nets are local in nature, we can employ the conventional placeand-route approach to obtain an optimized layout. The reason for cluster-level placement is that some clusters may have common macro-cells. In such a case, the common cells need to be placed at the common boundary. 


\section{EXPERIMENTAL RESULTS}

We present results for the following five benchmarks: (1) Compress; (2) Find; (3) FIFO; (4) TI's Shuffle Exchange Network; (5) Traffic Light Controller $(T L C)$. These are datapaths are automatically generated by a high level synthesis tool. Pertinent design data of the benchmark examples are shown in Table 1. We present the experimentation procedure below.

(1) Netlist preprocessing: From an input RTL netlist, power, ground, and clock nets are eliminated. Nets with identical cell lists are collapsed into one compound net, i.e., they are treated as a single bus. If two nets have no common cells then they are independent and the relative cell placement does not influence each other's total wirelength. In real designs, the degree of interdependency between two arbitrary nets lie between the above two extreme cases.

In a design consisting of $N$ number of nets, the node size of the net interdependency graph $(G)$ is equal to $N$ and the worst-case complexity of the number of edges is $\mathrm{O}\left(N^{2}\right)$. Therefore, the worst-case complexity of the number of nodes in $G_{d u a l}$ is $\mathrm{O}\left(N^{2}\right)$. In real life designs, the node-size of $G_{d u a l}$ is much smaller than the worst-case complexity. Column 3 of Table 1 reports the node-size of $G_{d u a l}$ for each design.

(2) Net Clustering: $G_{d u a l}$ is provided as an input to Tseng's clique partitioning heuristic [11]. The original algorithm proposed in [11] is modified to handle weighted input graphs. Optimal number of maximum weighted cliques are heuristically obtained. The nodes in the clique identify the cells that need to be in the same neighborhood. From the cliques and from the information about the cells associated with each node, we generate clusters of nets. A net may belong to more than one cluster, as the clique partitioning is performed on $G_{d u a l}$ rather than $G$. Recall that by doing so, not only do we find cliques of nets but also information as to which nets within the clique needs to routed closer. The latter information helps in prioritizing the nets when being routed as discussed in Section 3.. For each design, in Table 1 we tabulate the number of cliques obtained (column 5), the size of the largest clique (column 6), and the execution time for clique partitioning (column 7).

(3) Routing and Macro-cell Placement: The routing solution for each cluster is generated by hand. Work is in progress to integrate an interconnect synthesis tool that automatically generates the optimized network. We used Flint layout synthesis tool [17] in an interactive mode. We force Flint to generate a desired interconnect topology by generating appropriate channels in the placement. Flint has features to manipulate the macro-cells such as flipping, rotating etc., that are used extensively during the placement of macro-cells.

For each macro-cell, we generate standard cell layouts by employing "Stdcell" layout generator module in Lager silicon compiler. Thus, within each macro-cell cell placement is following by net routing. This approach is jus- 
tifiable as the nets within the macro-cell are short. From the MAGIC layouts generated by Flint, we measure the wirelength of each net. The nets are routed in two wiring layers (M1 and M2). We sum up the lengths of all M1 and M2 segments of a net to obtain its total wirelength.

\subsection{DISCUSSION OF RESULTS}

We demonstrate the efficacy of our approach in terms of the following design characteristics: (1) the sum of overall wirelengths of all nets in the design. (2) the bounding box area of the final layout. The reported values of the above measures are in the units of $\lambda$, the basic unit of scalable CMOS technology. All designs are targeted for static CMOS technology.

Below, we compare the optimized designs generated by route-and-place $(\mathrm{RnP})$ approach against those generated using Macro-cell (PnR) based layout style as well as Standard-cell (Stdcell) based layout style.

Total Wirelength. Table 2 summarizes the comparison of the total wirelength. The actual values of total wirelength are reported in columns 2-4. Columns 5 and 6 show the percentage change. In comparison with PnR, our approach yielded in significant wirelength reduction between $3.7 \%-24.6 \%$. This clearly demonstrates the effectiveness of the proposed approach. In comparison with Stdcell, our approach is not effective (but for Find) because of the following reasons: (1) Routing is limited to channels and hence in general the wirelength is higher. This can be alleviated by incorporating over-the-cell routing. (2) Macro-cell shape and port location information is not exploited. Work is underway to improve the approach by addressing these factors.

In Figure 5 we compare the results according the netlength distribution. In each figure, we plotted the net id on the $\mathrm{x}$-axis and its wirelength on $\mathrm{y}$ axis (in units of $\lambda$ ). The nets are sorted in ascending order according to their wirelength. As majority of nets in every design have reduced substantially, it is evident that the proposed methodology is highly effective. Equally important is the fact that there was no tradeoff between nets.

Bounding Box Area. The penalty paid for the wirelength reduction is an increase in the bounding box area. Table 3 summarizes the comparison of the bounding box area. The measured values are reported in columns 2-4. Columns 5 and 6 show the percentage change. In comparison with PnR, our approach resulted in an average increase of $25.8 \%$. In comparison with Stdcell, our approach resulted in an average increase of $109.2 \%$ !! But, this can be improved by incorporating area in the cost function while generating routing solution for a cluster. Compaction algorithms and over-the-cell routing algorithms will be integrated into the design flow to minimize the area penalty. 
Table 1 Pertinent design data and clique partitioning related data

\begin{tabular}{|l|c|c|c|c|c|c|}
\hline Design & Macro-cells & Nets & $\begin{array}{c}\text { Nodes } \\
\text { in } G_{\text {dual }}\end{array}$ & $\begin{array}{c}\text { No. of } \\
\text { Cliques }\end{array}$ & $\begin{array}{c}\text { Size of } \\
\text { Largest } \\
\text { Clique }\end{array}$ & $\begin{array}{c}\text { Exec } \\
\text { Time } \\
\text { seconds }\end{array}$ \\
\hline Compress & 35 & 106 & 62 & 14 & 8 & 0.39 \\
Find & 58 & 125 & 130 & 18 & 15 & 3.47 \\
Fifo & 63 & 91 & 179 & 33 & 20 & 3.48 \\
Shuffle & 104 & 43 & 353 & 37 & 67 & 52.57 \\
TLC & 32 & 30 & 86 & 19 & 10 & 0.42 \\
\hline
\end{tabular}

Table 2 Total Wirelength ("-"” stands for reduction)

\begin{tabular}{|l|r|r|r|r|r|}
\hline Circuit & PnR & RnP & Stdcell & vs. PnR & vs. Stdcell \\
\hline Compress & 343064 & 330536 & 100122 & $-3.7 \%$ & $+230.0 \%$ \\
Fifo & 1000242 & 754083 & 380351 & $-24.6 \%$ & $+98 \%$ \\
Find & 105256 & 99956 & 399962 & $-5.0 \%$ & $-75.0 \%$ \\
Shuffle & 1115374 & 873600 & 164289 & $-21.7 \%$ & $+432.0 \%$ \\
TLC & 129122 & 120004 & 40120 & $-7.0 \%$ & $+199.0 \%$ \\
\hline
\end{tabular}

Table 3 Bounding box area

\begin{tabular}{|l|r|r|r|r|r|}
\hline Circuit & PnR & RnP & Stdcell & vs. PnR & vs. Stdcell \\
\hline Compress & $2782 \times 2894$ & $3071 \times 3435$ & $2459 \times 1648$ & $+31 \%$ & $+160.3 \%$ \\
Fifo & $5076 \times 4583$ & $3368 \times 7529$ & $5735 \times 2832$ & $+9 \%$ & $+56.0 \%$ \\
Find & $4520 \times 5109$ & $4099 \times 6814$ & $5195 \times 2528$ & $+21 \%$ & $+112.7 \%$ \\
Shuffle & $6227 \times 4916$ & $4883 \times 6973$ & $5339 \times 3584$ & $+11 \%$ & $+77.9 \%$ \\
TLC & $1963 \times 1983$ & $2450 \times 2489$ & $1875 \times 1360$ & $+57 \%$ & $+139.1 \%$ \\
\hline
\end{tabular}

\section{CONCLUSIONS}

We introduced a novel route-and-place design methodology that is applied for interconnect minimization in DSM regime. Full flexibility to design the global nets is the key to high performance design in DSM regime. Extensions to this work includes integrating an interconnect synthesis tool, incorporating area minimization, and delay measurement using DSM-accurate timing tools.

\section{Acknowledgments}

We are thankful to Meenakshi Kaul and Ranga Vemuri at the University of Cincinnati for providing us with their clique partitioning implementation and the synthesis benchmarks. 
Fto

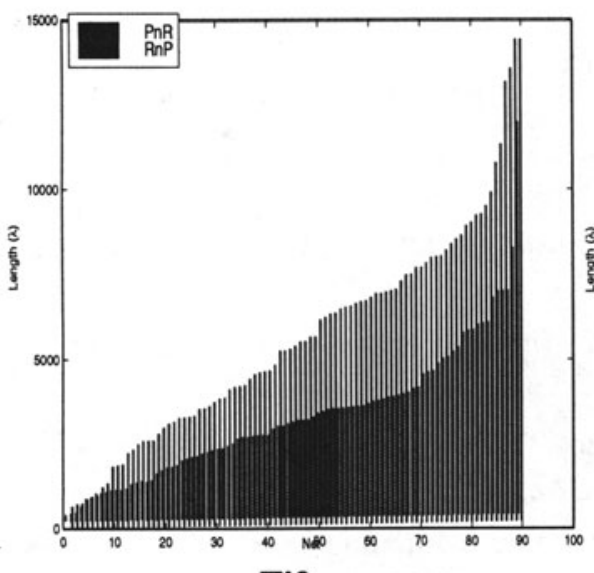

Fifo

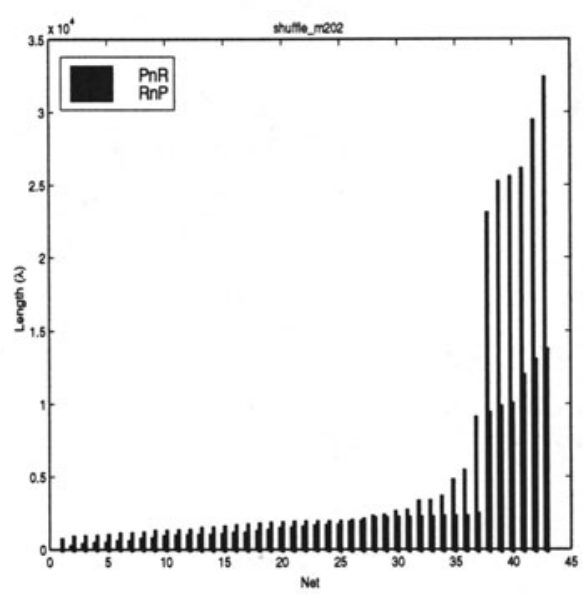

Shuffle

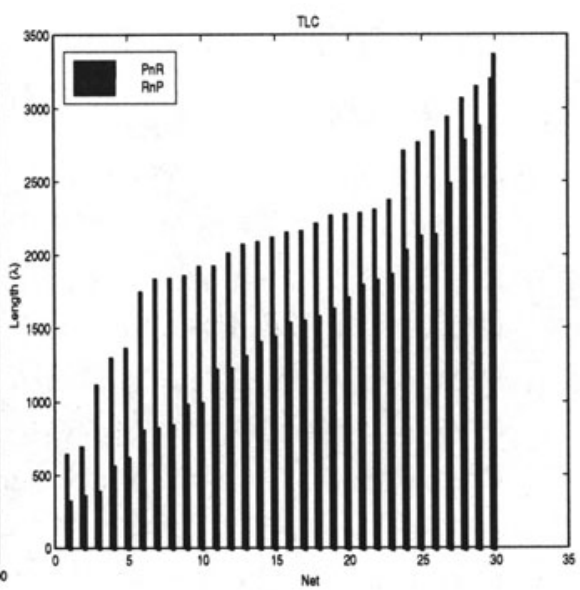

Tle

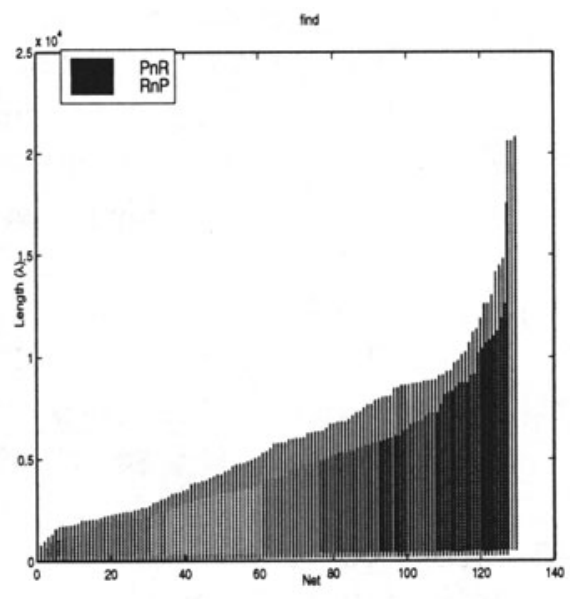

Find

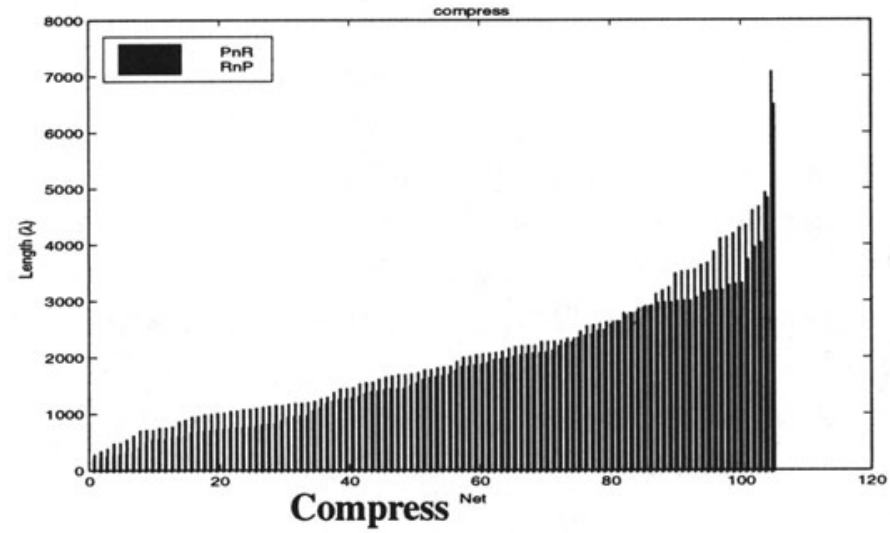

Figure 5 Comparision of the Wirelength Distribution 


\section{REFERENCES}

[1] -. "The National Technology Roadmap for Semiconductors". SIA (Semiconductor Industry Association), 1997.

[2] Pileggi L. "Delay metrics". In ISPD, 1998.

[3] R. H. J. M. Otten and R. K. Brayton. "Planning for Performance". In $D A C$, pages 122-127, June 1998.

[4] Sechen C. et. al. "The TimberWolf placement and routing package". In Proc. Custom Integrated Circuits Conference, May 1984.

[5] Breuer M. A. "Min-cut Placement". In J. Design Automation and FaultTolerant Computing, pages 343-382, 1977.

[6] Kernighan B. Dunlop A. "A procedure for placement of standard-cell VLSI circuits". IEEE Trans. CAD, CAD-4:92-98, 1985. Jan.

[7] Chaudhary K et. al. "RITUAL: Performance-Driven Placement of CellBased ICs". In Proc. 3rd Physical Design Workshop, May 1991.

[8] C-K Cheng J. Huang, X-L Hong and E.S. Kuh. "An Efficient Timing Driven Global Routing Algorithm". In DAC, pages 596-600, 1993.

[9] Dunlop A.E. et. al. "Chip layout optimization using critical path weighting”. In $D A C$, pages 133-136, 1984.

[10] Jackson M.A.B and Kuh E. S. "Performance-driven placement of cell based IC's". In DAC, pages 370 - 375, 1989.

[11] C. Tseng and D. P. Siewiorek. "Facet : A Procedure for the Automated Synthesis of Digital Systems". In 20th ACM/IEEE DAC, pages 490-496, 1983.

[12] J.Cong et. al. Performance-driven interconnect design based on distributed RC delay model. In $D A C, 1993$.

[13] A.B. Kahng et. al. Interconnect tuning strategies for high-performance ics. 1997.

[14] J. Cong et. al. Performance optimization of vlsi interconnect layout. In Integration, the VLSI Journal, pages 1-94. 1996.

[15] B.Tutuianu, et. al. An explicit rc-circuit delay approximation based on the first three moments of the impulse response. In $D A C$, pages $611-616$, 1996.

[16] Electronic Products. "Novel IC Shuffles Parallel Processing Data". pages 42-50, August 1986.

[17] R. W. Broderson, editor. Anatomy of a Silicon Compiler. Kluwer Academic Publishers, 1992. 